\title{
La invisibilización de las mujeres en la academia
}

\section{The invisibility of women in the academy \\ A invisibilização das mulheres no campo acadêmico}

\author{
Angie Vanessa Medina Zapata \\ Psicóloga \\ Universidad Surcolombiana \\ vameza0730@hotmail.com
}

\section{Resumen}

Mediante este ensayo cuestiono que la psicología, especialmente, pese a constituir un campo feminizado a nivel educativo y profesional, se caracteriza por prácticas de segregación territorial y jerárquica por sexos. En esta disciplina, como en muchas otras, (de las cuales menciono tres: literatura, ciencia y educación) el patriarcado invisibiliza las aportaciones históricas de las mujeres y las margina de sus merecidos reconocimientos. Argumentaré que al ser la psicología una disciplina y profesión mayoritariamente cultivada por mujeres, el hecho de que sean hombres quienes figuran como protagonistas en el mundo académico, no tiene otra explicación que un sesgo de género o, más exactamente, una discriminación sexista que desconoce el trabajo femenino en este campo.

Es pertinente aclarar que mi objetivo no es polarizar la situación y caer en otro "ismo". No se trata de negar las aportaciones de los hombres, no es una batalla entre el feminismo y machismo o una postura victimizadora; por el contrario, la finalidad de este ensayo es visibilizar las aportaciones de las mujeres que han sido consideradas por el sistema denominado patriarcado como secundarias a lo largo de la historia o no se han reconocido en absoluto.

Palabras clave: Patriarcado, sesgo de género, discriminación sexista, mundo académico.

\section{Abstract}

Through this essay, I question that psychology, especially, despite constituting a feminized field at an educational and professional level is characterized by practices of territorial and hierarchical segregation by sex. In this discipline, as in many others, (of which I mention three: literature, science, and education) patriarchy makes the historical contributions of women invisible and marginalizes them from their well-deserved recognition. I will argue that since psychology is a discipline and profession mainly cultivated by women, the fact that men are the protagonists in the academic world, has no other explanation than a gender bias or, more exactly, sexist discrimination that ignores the female work in this field. 
It is pertinent to clarify that my objective is not to polarize the situation and fall into another "ism". It is not about denying the contributions of men, it is not a battle between feminism and machismo or a victimizing position; On the contrary, the purpose of this essay is to make visible the contributions of women that have been considered by the system as patriarchy as secondary throughout history or have not been recognized at all.

Keywords: Patriarchy, gender bias, sexist discrimination, history, academic world.

\section{Resumo}

Através desse ensaio faço uma crítica à psicologia, embora seja um campo feminizado tanto na formação educativa quanto na profissional, caracteriza- se pelas práticas de segregação territorial e hierárquica dos sexos. Nessa disciplina, semelhante com muitas outras, por exemplo: literatura, ciência e educação, o patriarcado invisibiliza os aportes históricos das mulheres e as margina dos seus merecidos reconhecimentos. Desse modo, argumentarei que a psicologia é uma disciplina e profissão mormente cultivada por mulheres, entrentanto, o fato de que sejam homens os que comparecem como os protagonistas no mundo acadêmico, não tem outra explicação que um viés de gênero ou, mais exatamente, uma discriminação sexista que desconhece o trabalho feminino neste campo.

É pertinente afirmar que o meu objetivo não é polarizar a discussão e cair em mais um "ismo". Não procuro negar os aportes dos homens, nem aprofundar em uma batalha entre feminismo e machismo, ou um posicionamento de vítima; ao contrário, a finalidade desse ensaio é dar visibilidade aos aportes das mulheres que têm sido classificadas, pelo sistema denominado de Patriarcado, como secundárias ao longo da história e não tem recebido reconhecimento nenhum.

Palavras-chave: Patriarcado, viés de género, discriminação sexista, campo acadêmico.

No deseo que las mujeres tengan poder sobre los hombres, sino sobre sí mismas.

\section{Mary Wollstonecraft}

Para sustentar la afirmación de que existe invisibilización de las mujeres en el mundo académico, empezaré con una breve reseña histórica acerca de las contribuciones que han hecho las mujeres en la historia de la humanidad, en la psicología, pero no sólo en esta disciplina, también en la literatura, la ciencia y la educación. Esto con el fin de comprender las relaciones de género y el contexto histórico cultural en el que ocurren, para vislumbrar el sexismo en la academia (en general) a lo largo de los años. Luego, resaltaré la importancia que ha tenido el feminismo en la psicología y en otras disciplinas.

El feminismo como una herramienta que ha posibilitado reflexionar de forma crítica sobre las relaciones de poder vinculadas a la naturalización de la diferencia sexual, como una categoría que exige para la mujer el reconocimiento de unas capacidades y unos derechos que tradicionalmente han estado reservados para los hombres. (Rubin, 1996)

Pasaré luego a una revisión de la epistemología feminista, la cual enfatiza la importancia de los valores éticos y políticos en la conformación de las prácticas epistémicas y en las interpretaciones de evidencia. Es decir, estudia cómo el género influye en nuestra manera de entender el conocimiento, su justificación, y la teoría del conocimiento. Además, describe la manera en que se genera conocimiento y cómo su justificación pone a las mujeres en desventaja - respecto de los varones. 
Las epistemólogas feministas sostienen que la tradición científica discrimina a las mujeres. Por un lado, impidiéndoles investigar, y por el otro, presentando a las mujeres como inferiores. Las actuales teorías de conocimiento satisfacen sólo intereses masculinos, fortaleciendo así las jerarquías de género (Anderson, 2004).

Complementaré este concepto con los planteamientos de Kohlberg (1981) y Gilligan (1982), para establecer ciertas diferencias epistemológicas e investigativas en el desarrollo moral diferenciado entre hombres y mujeres y al mismo tiempo destacar "la ética del cuidado". Después, hablaré acerca de la posible influencia de la educación y los medios de comunicación en el sexismo académico y su relación con la supremacía de hombres y la difusión de una visión masculina del mundo.

Por supuesto, en cada punto que he mencionado se encuentra el contexto sociopolítico, pero profundizaré un poco más en este esperando que sirva de marco para entender e interpretar una serie de circunstancias y episodios relacionados con la invisibilización de la mujer en la academia y en la vida misma.

Finalmente, plantearé unas conclusiones a partir de lo investigado, recogido, reflexionado $\mathrm{y}$ vivido, porque indudablemente es un tema que me cruza por ser mujer, por tener unas vivencias particulares y por empatizar con otras experiencias colectivas tales como pertenecer a un proceso organizativo feminista. Dicho proceso, cuestiona nuestra situación de subordinación y en él se emprenden acciones para transformar y contribuir a la construcción de una sociedad más equitativa entre hombres y mujeres.

Como diría Joan Kelly-Gadol, el objetivo de este recuento histórico no es únicamente "devolver las mujeres a la historia" sino, sobre todo, "devolver la historia a las mujeres". Es muy importante que la población femenina conozca y reconozca su propia historia, la de sus experiencias en ella y de ella, una historia de las mujeres en cuanto mujeres, pero es de igual forma importante que esa experiencia, que las actividades que han realizado y que los espacios que han ocupado sean considerados de interés para la historia, puesto que ellas forman parte de los grupos sociales que articulan y mueven cada sociedad.

A lo largo de la historia han sido muchos los psicólogos que han influido en el desarrollo de la ciencia de la mente y el comportamiento humano. Es habitual hablar de Watson, Skinner, Bandura, Freud, Rogers, Maslow, entre otros, la gran mayoría hombres. Infortunada- mente, la voz de la mujer ha sido silenciada durante muchos años, y sus contribuciones fueron minimizadas o excluidas de los círculos científicos. Hoy en día parece imposible pensar que la psicología fue una profesión exclusivamente de hombres, porque en la actualidad es una carrera que estudiamos más mujeres que hombres. Como afirma Corbin (2014):

Lo cierto es que la psicología estaba considerada un terreno masculino, y las mujeres que deseaban labrarse un futuro profesional como psicólogas tuvieron que hacerse a un lado en una disciplina que sólo aceptaba hombres. Por suerte, los cambios sociales y económicos de este último siglo han permitido el crecimiento de la "psicología femenina". Igual que en otros campos, las mujeres han luchado para obtener los mismos derechos que los hombres.

Las mujeres han hecho contribuciones importantes e innovadoras en todos los campos. Estas, al igual que los hombres, merecen ser reconocidas por su trabajo pionero. En primer lugar, se encuentra Crhistine Ladd- Franklin, ella tenía intereses que incluían la psicología, la lógica, las matemáticas, la física y la astronomía.

En 1929 desafió uno de los psicólogos varones importantes de la época, Edward Titchener, por no permitir a las mujeres entrar en su grupo de experimentadores y desarrolló una influyente 
teoría de la visión del color. Al igual que su madre y su tía fue firme partidaria de los derechos de las mujeres. Esta temprana influencia no sólo la ayudó a tener éxito en su campo, a pesar de la considerable oposición, sino que también inspiró su obra posterior sobre la defensa de los derechos de las mujeres en el mundo académico.

En la psicología, por ejemplo, cuando la mayoría de la gente habla de Anna Freud, la relaciona directamente con Sigmund, el padre del psicoanálisis. Sin embargo, la hija del famoso psicoanalista fue una psicóloga muy conocida e influyente por derecho propio.

Anna Freud no sólo amplió las ideas de su padre, sino que también desarrolló el campo del psicoanálisis de la infancia e influyó en otros pensadores incluidos Erik Erikson. Entre sus muchos logros está la introducción de los mecanismos de defensa, esto llevado a cabo en 1945.

Por otra parte, se encuentra Eleanor Maccoby , quien realizó grandes aportes en la psicología del desarrollo. Su trabajo fue pionero en la psicología y las diferencias de sexo, jugando un importante papel en la comprensión sobre temas como la socialización, las influencias biológicas en las diferencias de sexo y los roles de género. Ella fue la primera mujer en presidir el departamento de psicología de la Universidad de Stanford y la primera mujer en realizar sus conferencias llevando un traje pantalón hacia el año 1966, década en la cual se imponía una hiperfeminización en la vestimenta de la mujer, que buscaba potenciar su rol tradicional de madre y esposa; por lo tanto, se convierte en un acto revolucionario.

Otras mujeres que aportaron a la historia de la psicología y no fueron reconocidas son Leta Stetter Hollingworth, hizo contribuciones significativas (1916) en tres áreas. En primer lugar, trabajó en la psicología de la mujer, contrastó experimental- mente algunas de las diferencias sexuales a las que se apelaba para limitar las posibilidades intelectuales $\mathrm{y}$ profesionales de las mujeres. $\mathrm{Su}$ segunda área fue la psicología clínica, su tesis doctoral comparaba las habilidades motoras y mentales de las mujeres durante el periodo menstrual y fuera de él; hizo pruebas semejantes a los hombres y no encontró evidencia de que las mujeres tuviéramos un ciclo de debilitamiento, como se sostenía. En tercer lugar, se dedicó a la psicología educativa, consideraba que los factores educativos y ambientales también desempeñaban un papel clave en el desarrollo del ser humano. Por ello, se interesó en cómo asesorar y educar adecuadamente a las personas superdotadas, además, Carl Rogers fue su alumno.

En contraste, se encuentra Karen Horney, que en el año 1937 cuestionó mediante sus teorías ciertos puntos tradicionales del pensamiento de Sigmund Freud. Por ejemplo, que las diferencias psicológicas entre el hombre y la mujer no son producto inherente de la biología humana, sino que deben su origen a diversos factores culturales y sociales. Su pensamiento es clasificado dentro del neofreudismo.

Mamie Phipps Clark, psicóloga experimental, es la precursora del famoso experimento de los muñecos de color negro y blanco llevado a cabo en 1940, en el cual se evidenció en los niños y niñas el interés por los muñecos de color blanco ya que, según ellos, los muñecos negros eran "feos". Por lo tanto, plantea que posiblemente ellos y ellas muestran mayor atracción por los estereotipos de color blanco ya que son más aceptados por la cultura. Es reconocida por sus investigaciones sobre la discriminación y su liderazgo en el campo del feminismo, además de su amplio conocimiento sobre la cultura afrodescendiente.

Melanie Klein, psicoanalista austriaca creadora de una teoría del funcionamiento psíquico hizo importantes contribuciones entre 1924 y 1957 sobre el desarrollo infantil desde la teoría psicoanalítica y fundó la escuela inglesa de psicoanálisis. Alice Miller, es una psicoanalista polaca conocida por su trabajo (1953-1986) en maltrato infantil y sus efectos en la sociedad, así 
como en la vida de los individuos. Aunque es pertinente resaltar que no sólo estas mujeres han hecho aportaciones, quedan muchas más sin mencionar.

Pasando al ámbito de la literatura se encuentra Christine de Pizán, la cual se convierte en la primera escritora profesional que venció numerosos obstáculos, comprobó en directo la marginación de la mujer y, como reacción a la misoginia que proclamaban tantos libros, escribió uno de los primeros alegatos feministas, La ciudad de las damas (1405). Está también Sor Juana Inés de la Cruz, autora de innumerables poemas que la llevaron a ser reconocida como una de las exponentes más importantes de la literatura hispanoamericana del Siglo de Oro (1492-1681). Fue una artista que revolucionó la forma de pensar y cuestionó las convencionalidades de una sociedad donde el conocimiento y el anhelo por aprender eras destinados exclusivamente a los hombres. Abordó un sinfín de temáticas que no eran estudiadas por las monjas de la época, tales como el amor y el desamor, lo regligioso y lo profano, pero también la sátira y la comedia.

Por contraste, Oriana Fallaci publicó un diario que llevó mientras cubrió la guerra de Vietnam. Ella fue una escritora, periodista y activista italiana, la primera mujer de su país corresponsal de guerra. Las primeras obras de su autoría son Los siete pecados capitales de Hollywood (1959), El sexo inútil (1961) y Penélope en la guerra (1962). De igual forma, hacia 1972, Gioconda Belli, poeta nicaragüense, publicó sus primeros textos y de inmediato generó polémica debido a la manera en que abordaba el cuerpo y la sensualidad femenina.

"¿Qué necesitan las mujeres para escribir buenas novelas? Independencia económica y personal: ¡UNA HABITACIÓN PROPIA! Tras esta premisa se esconde la reflexión de la novelista, ensayista, editora, feminista y cuentista británica, Virginia Woolf" (Farianas, 2017, pp. 2). Según ella, la literatura no sirve solo para contar historias: también nos representa, nos cuestiona y nos ayuda a pensarnos como individuos y como integrantes de la sociedad.

¿Ese es el panorama real de la literatura? ¿Sin voces femeninas? No está ni María Teresa León, María Martínez Sierra, Maribel Lázaro, Ana Diosdado, ni Paloma Pedrero. O, en poesía, Concha Méndez, Carmen Conde, María Victoria Atencia, Olvido García Valdés, Gloria Fuertes, Ana Rossetti, ni Chantal Maillard, por citar apenas algunas de las numerosísimas que existen. ¿Por qué nos hurtan esas voces? $\mathrm{Y}$, sobre todo, ¿qué respuesta hemos dado a esta ausencia? (Arroyo, 2013, El país)

Haciendo énfasis ahora en la ciencia recuerdo una lectura de El Comercio, un diario que se publica en Perú en donde mencionaba que hacía unos pocos días se celebraba en el mundo el Día Internacional de las Mujeres y Niñas en Ciencia, promovido por la UNESCO. Pero que en realidad no era un día para celebrar porque, pese a los extraordinarios avances de las ciencias y las tecnologías, en diferentes disciplinas, la ciencia mantiene un estatus sexista.

Aunque en la misma columna el autor, Fausto Segovia, mencionaba que hay esperanzas ya que la adopción de la Agenda 2030 para el Desarrollo Sostenible centrada en la igualdad de género representó una victoria importante para quienes defienden las inversiones y políticas financieras, económicas, ambientales y sociales, incluida ONU Mujeres. Es que no es posible un desarrollo sostenible si a la mitad de la humanidad se le sigue negando la plenitud de sus derechos humanos y oportunidades.

En una ocasión dijo Voltaire que había conocido muchas mujeres científicas muy inteligentes, pero ninguna inventora. Parecía así negarles capacidad inventiva, algo que se ha perpetuado en la falsa creencia de la incapacidad de las mujeres para la ingeniería. 
Los hechos históricos nos demuestran lo erróneo de esa idea: Josephine Cochran, inventora estadounidense del pri- mer lavavajillas comercialmente exitoso, su invento se expuso en 1893, en la Exposición Universal de Chicago y ganó el primer premio por la mejor construcción mecánica, duradera y adaptada al ritmo de trabajo.

Mary Anderson, nacida en Alabama, en noviembre de 1903 se le concedió su primera patente para un dispositivo automático de limpieza de la ventana de automóvil controlado desde el interior de este, llamado el limpiaparabrisas.

La microbióloga Elizabeth Lee Hazen y la química Rachel Fuller Brown descubrieron y aislaron en 1931, uno de los primeros fungicidas aplicables en humanos, la nistatina, lo hicieron gracias a su talento y perseverancia, pero también en parte gracias a la eficacia del servicio de correos estadounidense de la época.

Las mujeres en la NASA, un equipo de matemáticas conocidas como las computadoras humanas (Katherine Johnson, Dorothy Vaughan y Mary Jackson) calcularon, con lápices, reglas y sencillas calculadoras, las complicadas ecuaciones que permitieron lanzar los cohetes y a sus astronautas al espacio hacia el año 1959. A ellas se les atribuye la trayectoria del vuelo espacial de Alan Shepard, el primer estadounidense que viajó al espacio, y en 1961 la ventana de lanzamiento del Proyecto Mercury, el primer programa espacial tripulado de los EE UU. Gertrude Ellion, fue una bioquímica y farmacóloga estadounidense, que recibió en 1988 el Premio Nobel de Fisiología y Medicina por sus descubrimientos de los principios clave sobre el desarrollo y el tratamiento de medicamentos; son algunos ejemplos significativos.

Se suele señalar a Manuel Jalón, en 1964, como el inventor español de la fregona, pero Julia Montoussé Frages (de origen francés, aunque avilesina de adopción) y su hija Julia (Julita) Rodríguez-Montussé obtuvieron, en 1953, una patente muchos años antes que el mecánico de aviones. (Pérez, E; 2018)

Para cerrar la breve reseña histórica, es pertinente resaltar el papel de la educación y el fuerte impacto que generó el grupo femenino de la Generación del 27 en España. Lira, (2018) afirma:

Las artistas españolas del 27 reivindicaron su papel intelectual no sólo sobre su propia figura, sino sobre la vida cultural que las rodeaba. Aparecen en publicaciones de la época haciendo reseñas sobre libros, opinando sobre arquitectura y formando parte de una ajetreada agenda social. Eran escritoras, filósofas, escultoras, poetas, actrices e ilustradoras. Abiertas a nuevos conceptos de modernidad y a las corrientes de vanguardia que provenían de Europa. Profundamente comprometidas con su tiempo y su realidad social, su actitud fue rompedora y abierta, transformando el panorama cultural y artístico de una España convulsa. (p. 2)

A pesar de todo el talento que tenían, sus nombres se esfumaron. La historia, la educación y la literatura las olvidaron casi por completo. Si sumamos la "contrariedad" de ser mujer en aquella época a la del exilio, el resultado es la invisibilización. Renunciaron a tanto y escasas páginas apenas las mencionan.

Muchas de las anteriores mujeres mencionadas han tenido un vínculo estrecho con el feminismo. Fue a comienzos del siglo $\mathrm{XX}$, coincidiendo con el estallido del movimiento sufragista y con la incorporación de las mujeres a los estudios superiores, y gracias a figuras como Karen Horney y Stetter Hollingworth, cuando estalló una nueva revolución feminista a todos los niveles, es decir, el feminismo como movimiento político, como postura ética y como categoría de estudio. El feminismo va de una movilización social y política hasta la elaboración teórico-crítica que se traduce en praxis social y política (Braidotti, 2000; Posada, 1999). 
Como movimiento social, surge hacia finales del siglo XVIII, y se torna político en el siglo subsecuente, cuando las mujeres luchan por el derecho a emitir el voto. Previo a esto, ya habían ocurrido disputas acerca de la igualdad de los sexos en la Ilustración, a partir de los tratados sobre educación que surgieron durante el Renacimiento. La filosofía que acompaña la lucha de emancipación de las mujeres es el pensamiento liberal, mismo que produjo la formulación de los Derechos del Hombre y del Ciudadano, pero que no incluía a las mujeres, por lo que Olimpia de Gouges, en 1791, se dio a la tarea de presentar la versión femenina: los Derechos de la Mujer y la Ciudadana, lo que ocasionó que fuera guillotinada. En 1792, Mary Wollstonecraft (1792/1998) considera la necesidad de que las mujeres sean educadas y no domesticadas.

Como postura ética, "el género es una herramienta que posibilita reflexionar de forma crítica sobre las relaciones de poder vinculadas a la naturalización de la diferencia sexual, una categoría que posibilita comprender cómo representamos el mundo y cómo nos representamos en él" (Bonilla, V et al., 2014). Un ejemplo claro de esto son las creaciones de la poeta mexiquense Sor Juana Inés de la Cruz, pioneras en la proclamación del derecho a pensar y escribir sin importar la condición de género. Esta autora es difusora mediante sus obras de las desigualdades entre hombres y mujeres, por lo tanto, resalta la importancia que representan para la literatura universal.

El género es visto también como categoría de estudio analítica, flexible, dinámica y contextual, debido a que al reconocer que el estudio de los términos sexuados y sus interrelaciones ayuda a entender los nexos entre el individuo y la organización social.

Además, la dimensión política del género -aspecto central de la herramienta conceptual- pone de manifiesto que el estudio de los conceptos vinculados a la diferencia sexual posibilita comprender cómo incorporamos estructuras de pensamiento que modelan la auto-percepción, vida social y prácticas culturales dentro de un grupo humano. (Bonilla, 2014, p. 35).

Virginia Woolf lo demuestra en su obra Una habitación propia al incitar reflexiones sobre el género y al denunciar cómo éste ha limitado a las literatas. Así, se refiere a un factor que no se ha erradicado aún: la injusticia de que ciertos constructos sociales limiten el desarrollo de algunos individuos. Mujeres y hombres, en todos los ámbitos, y en su caso (el literario), merecen siempre las mismas oportunidades y derechos para poder explotar sus potenciales al máximo.

También fue en la ciencia psicológica donde las mujeres empezaron a investigarse y analizarse a ellas mismas y a sus homólogas para demostrar que no estaban condicionadas para siempre por su biología y que no todos nuestros malestares responden a las hormonas. Sino que, la gran mayoría de esos malestares, son fruto de esforzarnos y/o ser obligadas por la fuerza a cumplir unos mandatos de género impuestos, los cuales ni deseábamos. Leta Stetter Hollingworth centró su interés en desmentir la hipótesis de variabilidad, que afirmaba que las mujeres eran más parecidas entre sí que los hombres, que presentaban una gama mucho más amplia de talentos y defectos. Por ello, había más hombres genios y con deficiencias mentales y las mujeres nunca alcanzarían los logros más altos, conformándose con la mediocridad. Por ende, realizó un trabajo pionero en Psicología de las Mujeres, rompiendo la idea de su inferioridad intelectual, esencialmente durante la menstruación.

El pensamiento científico y racional moderno se ha construido sobre la base de metáforas de "mentes" y "razones" masculinas que conocían "naturalezas" femeninas (Keller, 1991), reforzando un pensamiento dicotómico que construía a la mujer-científica como una 
contradicción en sus propios términos (Rossiter, 1992). Frente a ello, desde el feminismo, se han realizado estudios pedagógicos sobre cómo socializar y enseñar una ciencia no-sexista, al tiempo que los estudios historiográficos han recuperado a mujeres científicas, a figuras "femeninas" olvidadas en los procesos de definición e historización de las disciplinas y, sobre todo, nos han narrado sus experiencias desiguales de opresión y resistencia marcadas por la diferencia sexual (González García y Pérez Sedeño, 2002). Es decir, el feminismo plantea una educación con perspectiva de género, así como políticas públicas que contribuyan a desarrollarla, para propiciar la creación de un nuevo horizonte cultural, el de la equidad.

Una de las aportaciones sustantivas de la reflexión teórica feminista a la ciencia, la literatura y la psicología ha sido la categoría de género, que ayuda a comprender las relaciones sociales entre hombres y mujeres y la manera en que la condición de unos y otras se construye por dichas relaciones en un contexto más amplio denominado "sistema sexo-género". (Rubin, G. 1989). Dicho sistema opera de manera cruzada con otros sistemas de relaciones sociales, como la producción económica, la nacionalidad, la religión o la educación, entre otros. El sistema sexo-género no sólo organiza las relaciones entre unos y otras, sino que construye lo que cada quien ha de ser en este juego de relaciones. También condiciona la valoración asimétrica en las relaciones intergenéricas, en las que los hombres detentan la posición dominante.

Complementando este sistema surge la epistemología feminista en respuesta a la tradición científica que se había desarrollado desde las experiencias y la visión del mundo de un personaje en concreto, es decir, un hombre blanco, de clase alta, heterosexual y occidental, inaugurando así, otras formas de conocer. Velasco (2009) sintetiza algunos de los objetivos de la epistemología feminista a partir del siguiente objetivo general: Desvelar y cuestionar las lógicas binarias de hombre-mujer, femenino-masculino, activo- pasivo, público-privado, racional- emocional.

Los estudios realizados por Gilligan son un buen reflejo de dicha epistemología. Esta psicóloga estadounidense criticó la teoría del desarrollo moral de Kohlberg por considerar que no representaba adecuadamente el razonamiento moral femenino.

Realizó un estudio en el cual los sujetos eran mujeres y los dilemas que se proponían eran cuestiones reales, como el aborto. El resultado fue el descubrimiento de un modelo ético diferente al propugnado por Kohlberg. Si éste respaldaba como modelo de desarrollo moral una ética de la justicia, en los estudios de Gilligan salió a la luz la ética del cuidado. (Marín, 1993).

Las investigaciones de Gilligan ponían de manifiesto que el problema primordial de los estudios de Kohlberg fue su restricción a sujetos masculinos, la cual incluye una desviación de los resultados impulsada por la diferencia en la educación vital y moral que recibimos las mujeres y los hombres en la sociedad. Nosotras actuaríamos pensando en lo contextual, produciendo así un predominio de la puesta en valor del bienestar de los demás, como resultado de los condicionantes sociopsicológicos que determinan nuestra manera de ser, estar y actuar en el mundo, teniendo una concepción global y no sólo normativa de la moral. Mientras que los hombres actuarían basados en lo formal $\mathrm{y}$ abstracto, es decir, poniendo la justicia $\mathrm{y}$ los derechos por encima de las cuestiones y de cuidado. Se podría afirmar que ambas posturas apuntan a la igualdad, sin embargo, la ética del cuidado pretende resaltar el respeto a la diversidad y se preocupa por la satisfacción de las necesidades del otro. En cambio, la ética de la justicia hace énfasis en la imparcialidad y la universalidad, lo que elimina las diferencias. Por lo tanto, es pertinente manifestar que la ética del cuidado entiende el mundo como una red de relaciones y lo importante no es el formalismo, sino el fondo de las cuestiones sobre las que hay que decidir. 
Un ejemplo clave en la propuesta de la epistemología feminista es el ataque a los estudios de género con la expresión peyorativa ideología de género. Los estudios e investigaciones de género que respaldaban la cartilla sobre la discriminación sexual en los colegios colombianos fueron satanizados y así se dividió al país. El documento atacado consta de 34 preguntas que les permite a los rectores establecer si los manuales escolares promueven o admiten conductas discriminatorias por razones de género, credo, condición social, raza o identidad sexual, entre otros.

Este documento de 97 páginas, producto de un convenio entre el MEN y el Pnud, el Unfpa y la Unicef (organismos de la ONU), surgió con el propósito de "brindar elementos para fomentar los procesos de sensibilización, reflexión y transformación de los imaginarios existentes, con respecto a los roles de género" en ambientes escolares. En dos de sus apartados propone, por ejemplo, deconstruir la idea de que solo existen dos sexos y abrir la posibilidad de entenderlos más allá de aquello que históricamente se nos ha enseñado y que se empieza a entender que no se nace siendo hombre o mujer, sino que se aprende a serlo, de acuerdo con la sociedad y época con la que se crezca. Planteamiento inadmisible para los moralistas y fanáticos religiosos.

Pero como lo manifiesta la directora de Colombia diversa, Marcela Sánchez, por una estrategia de desinformación se ha perdido el foco de lo importante y es la violencia y discriminación hacia todo lo que sea diferente en la escuela. Es por esto que algunas personas manifiestan que dichas cartillas presentan una sociedad sin diferencias de sexo y vacía el fundamento antropológico de la familia, y por eso puede terminar confundiendo a los niños.

Lo que ciertas personas moralistas no han podido entender es que la construcción de los contenidos expuestos en la cartilla se realizó desde la revisión conceptual y teórica de aspectos esenciales que constituyen la sexualidad autónoma y libre de las personas en el contexto educativo, así como la consulta de experiencias previas y exitosas del abordaje de las orientaciones sexuales e identidades de género no hegemónicas en la escuela lo cual causa polémica ya que representa salir de la zona de confort y dar paso a nuevas formas de educación y convivencia ciudadana; es decir, el rechazo a propuestas incluyentes, visibilizadoras y participativas.

Es pertinente también mencionar otra situación desoladora en el país, relacionada con la epistemología feminista, como lo fue el resultado del plebiscito y el llamado enfoque de género en el Acuerdo de Paz. Una vez más el término con el que fue tergiversado por los promotores del NO, especialmente por los sectores religiosos encabezados por el exprocurador Alejandro Ordóñez, fue "la ideología de género". Un concepto sobre el que giró el debate electoral del plebiscito del 2 de octubre de 2016 y que acusaba al Acuerdo de Paz de imponer un nuevo modelo de familia.

En los acuerdos de La Habana aparece con infinita intensidad. Ya no será la ideología de género impuesta a nuestros hijos (a través de las cartillas del Ministerio de Educación), sino que estará en la Constitución. El Gobierno y las FARC pretenden que la ideología de género sea norma constitucional, señalaba con vehemencia Ordóñez.

Estos estereotipos son el fiel reflejo de una sociedad retrógrada que ha puesto una resistencia a una educación como la mencionó García Márquez (1982):

Una educación inconforme y reflexiva, que nos inspire un nuevo modo de pensar y nos incite a descubrir quiénes somos en una sociedad que se quiera así misma...Que canalice hacia la vida la inmensa energía creadora que durante siglos hemos despilfarrado en la depredación y la violencia, y nos abra al fin la segunda 
oportunidad sobre la tierra que no tuvo la estirpe desgraciada del coronel Aureliano Buendía. Por el país próspero y justo que soñamos: al alcance de los niños.

Para concluir, haré énfasis en tres aspectos. En primer lugar, es pertinente denunciar la jerarquía social de desvalorización que, frente a los hombres y en el contexto patriarcal, nos ha acompañado a las mujeres a lo largo de la historia; la exclusión, la discriminación, el silenciamiento, la omisión, los sesgos, la devaluación, especialmente de lo femenino y de nosotras mismas, que posteriormente incorporará otras posiciones históricamente vulnerabilizadas. Tal como ha ocurrido con la vida académica de las mujeres. Ante esto, considero que la epistemología feminista, junto con la perspectiva de género, se constituyen como opciones frente a las premisas biologicistas y esencialistas que establecen o naturalizan diferencias por sexo, raza, discapacidad, y las premisas universalistas y coloniales que tienden a la homogeneización de los cuerpos y las experiencias.

En segundo lugar, es evidente la influencia de los medios de comunicación en el sexismo académico. El poder se define en los distintos discursos desde la posición de fuerza y control de los personajes masculinos que aparecen.

La perspectiva de género se hace así también necesaria en el tratamiento informativo de la violencia de género, puesto que no solo nuevos conceptos, sino también nuevos enfoques surgen a la hora de representar la realidad en los medios. Los medios de comunicación tienen una responsabilidad de mediación entre los sucesos y los espectadores $\mathrm{y}$, al parecer, este vínculo filtra ciertos aspectos de la realidad, reproduciendo el machismo y asentando como realidad incontrarrestable la inequidad y el detrimento de las mujeres en relación con los hombres.

Finalmente, no se trata de "devolver las mujeres a la historia" sino, sobre todo, "devolver la historia a las mujeres" ya que el sistema patriarcal ha podido funcionar debido a imposiciones ejercidas sobre las mujeres. Lo anterior viene avalado de varias maneras: la inculcación de los géneros; la privación de la enseñanza; la prohibición a las mujeres para conocer nuestra propia historia; la división entre nosotras al definir la respetabilidad y la desviación a partir de nuestras actividades sexuales; mediante la represión y la coerción total, por medio de la discriminación en el acceso a los recursos económicos, académicos y el poder político; y al recompensar con "privilegios de clase" a las mujeres que se conforman. No se trata entonces de plantear conclusiones tajantes, sino que las mujeres encontremos, al fin, nuestro espacio propio en la historia y, por ende, en la academia.

\section{Referencias}

Así va el enfoque de género en el posconflicto. (2017) Gómez, P. El Espectador. Colombia.

Blanes, P., 2015, "Olvidadas y silenciadas. Las mujeres de la generación del 27 que debes conocer", SER. Disponible en: http://cadenaser.com/program a/2015/10/ 08/la_script/14442 88990_684693.html (3 de octubre de 2017).

Bonilla, V. (2014). Una sola carne: deseo, amor y región. Estudio de caso en el Huila. Tesis de maestría. Bogotá: Universidad Nacional de Colombia.

Bourdie, P. (2011). Las estrategias de la reproducción social. México: Siglo XXI.

Bueno Ballesteros, A., 2016, "Lo que aprendí de las mujeres de la Generación del 27 viendo 'Las Sinsombrero", Verne.

Castellanos, G. (2003). Sexo, género y feminismo: tres categorías en pugna. En: P. Tovar (Edit.). Familia, género y antropología. Desafíos y transformaciones (pp. 30-65). 
Bogotá: Instituto Colombiano de Antropología e Historia.

Pérez, E. (2018) 11 de febrero: Un día para rescatar a nuestras inventoras del olvido.

Rubin, G. (1989). Reflexionando sobre el sexo: notas para una teoría radical de la sexualidad. En: Carole Vance (Comp.). Placer y Peligro. Explorando la Sexualidad Femenina (pp. 113 - 190). Madrid: La Revolución.

Velasco, Sara (2009). Sexo, género y salud. Madrid: Minerva. 